\title{
Performance und Theater als anderes Sprechen
}

\author{
Wolfgang Sting
}

\begin{abstract}
Zusammenfassung
Die aktuellen performativen Spiel- und Inszenierungsformen der szenischen Künste halten innovative Impulse für die Konzeption und Praxis des Sprachenlernens bereit. Das zeigen die Kategorien des Performativen, die am Beispiel des Flashmobs in diesem Beitrag herausgearbeitet werden wie z.B. Aktion, Körperlichkeit, Bewegung, Intensität, Gemeinschaft, Spielfreude, Ereignischarakter, also Improvisieren, Inszenieren, Präsentieren und soziale Interaktion im öffentlichen Raum. Diese körperlichen, sozialen und ästhetischen Handlungs- und Erfahrungskategorien markieren wesentliche Elemente jugendlicher Lebenswelt und unterstützen Lernprozesse nachweislich. Performative Praxis bedeutet Wirklichkeit und Wirkung erschaffen; didaktisch gewendet heißt das: Performance und Theater allgemein als Spiel- und Interaktionsform leben von einer elementaren Handlungs-, Erfahrungs-, Subjekt-, Gruppen- und Situationsorientierung. Deshalb sind pädagogische Settings und Lernkonzepte heutzutage sehr an Performance und dem Moment der Performativität interessiert. Im diesem Beitrag wird am Beispiel des TheaterSprachCamps in Hamburg illustriert, welchen Stellenwert eine am Performativen orientierte Theaterpraxis in diesem Konzept der Sprachförderung einnimmt und welche Wirkung sie entfaltet. Sprechen an dieser Stelle verweist darauf, dass Theater und Performance als Kunst- und Kommunikationsform neben Sprache als gesprochenem Wort eine Vielzahl von Sprachen und Sprechformen wie etwa Körper-, Bild-, Symbol-, Rhythmus-, Bewegungs, Raumsprache bzw. szenisches und performatives Sprechen umfasst. Der Beitrag basiert auf schon veröffentlichten Passagen aus Sting (2010), Sting (2011), siehe Literaturverzeichnis.
\end{abstract}

\section{Einleitung}

Performance ist ein Schlüsselbegriff des 21. Jahrhunderts, so der amerikanische Medien- und Performancewissenschaftler Jon McKenzie in seinem Buch „Perform or Else“ (2001). Interessant ist dabei seine These: Performance habe den Begriff der Disziplin, diesen Inbegriff der bürgerlicher Emanzipation aus dem 19. und 20. Jahrhundert abgelöst. Nicht ob man diszipliniert Normen und Werte einhalte, schaffe soziale Akzeptanz, Status und Macht, sondern, ob und dass man sich erfolgreich präsentieren könne. Da fallen uns doch gleich einige 
Personen ein, die sich immerfort präsentieren, auch ganz erfolgreich, aber gar nichts geleistet haben im Sinne protestantischer Arbeitsethik: wie etwa Paris Hilton und andere Sternchen oder auch plagiatsüberführte Politikerfiguren, die sich im Rampenlicht gekonnt inszenieren. Performance ist aber nicht nur in der Glamourwelt, sondern auch in Politik, Business, Sport und Alltag angesagt, um sich zu zeigen und zu vermarkten. Mit dem Begriff Performance kann somit die öffentliche Inszenierung und Darstellung, aber auch eine Leistung gemeint sein.

Einen gekonnten Auftritt hinzulegen erfordert immerhin ein reflektiertes Verständnis von Inszenierungsformen und -strategien. Performance als inszeniertes Wirken und Handeln zu beherrschen, ob medienwirksam, künstlerisch oder im pädagogischen Setting, ist deshalb im Bereich ästhetisch-kultureller Praxis und Kompetenz anzusiedeln und verdient eine intensivere Betrachtung.

Meine These ist, dass Performance wie Theater als Handlung- und Kommunikationsform vielfältige szenische, sinnlich-körperliche und wirklichkeitskonstituierende Lernchancen für Sprachlernen eröffnen. Performance ist eben mehr als nur Spiel und Darstellung (als-ob), sondern ganz unmittelbares performatives Tun und Sprechen.

Mein Beitrag gliedert sich in zwei Teile. Erstens möchte ich Merkmale und Qualitäten von Performance und performativem Handeln aufzeigen. Zweitens möchte ich am Beispiel des TheaterSprachCamps in Hamburg herausstellen, welchen Stellenwert performative Theaterarbeit in diesem Konzept der Sprachförderung hat.

\section{Performative Praxis am Beispiel Flashmob}

Zum Einstieg möchte ich den sogenannten Flashmob als ein Beispiel performativer Praxis vorstellen Ein Flashmob, übersetzt Blitzauflauf, ist eine öffentliche Aktion, zu der sich fremde Menschen im Internet oder über Handy verabreden. Dabei gibt es ganz unterschiedliche soziale und ästhetische Formate und Anlässe, Orte und Gruppengrößen. Hans Magnus Enzensberger hat schon 1987 in seinem Essayband „Ach Europa“ das Phänomen blitzartig auftauchender Jugendlicher in Schweden, die sich damals noch übers Telefon organisierten, als politische Unruhe-Aktion beschrieben. Beim Flashmob werden für einen meist kurzen Zeitraum ungewöhnliche Handlungen an alltäglichen Orten wie Straßen, Plätzen, Kaufhäusern aufgeführt. Hier nur eine kleine Auswahl beispielhafter Aktionsformen: Eine Gruppe von 40 Fahrradfahrer/inne/n fährt in einen Lübecker Kreisverkehr und bildet einen geschlossenen Kreis, so dass kein Auto mehr aus dem oder in den Kreisverkehr fahren kann, nach zwei Minuten löst sich alles auf. 4.500 Jugendliche überfluten e ine Münchner McDonald-Filiale und bestellen alle das gleiche, der Betrieb bricht zusammen. Hunderte Menschen liefern sich fünf Minuten lang eine Massen-Kissenschlacht vor dem Kölner Dom. Oder vielfältige Freeze Aktionen, eine kleine Gruppe friert im Supermarkt zwei Minuten zu Statuen ein bis hin zum großen „Freeze Flashmob in Paris" wo Hunderte eine fixierte Position halten. Daneben gibt es 
auch politisch motivierte Aktionen, Smartmobs genannt.

Meist werden die Flashmobs gefilmt und anschließend ins Internet gestellt. Bei „YouTube“ (www . youtube .com) kann unter dem Stichwort „Flashmob“ eine Vielzahl dieser durchaus unterhaltsamen Blitzaktionen aus aller Welt angeschaut werden. So wird die öffentliche Aktion auch noch der Netzöffentlichkeit vorgestellt. Die kurzfristige Intervention wird dokumentiert, die Reaktionen der Zuschauenden und die Veränderung der öffentlichen „Ordnung“ ausgestellt, mitunter auch szenisch gestaltet mit Schnitt und Musik.

Ein Flashmob ist auf den ersten Blick oberflächlich, spaßorientiert, aktionistisch, gruppenbestimmt, kurzzeitig und intensiv. Auf den zweiten Blick zeichnet sich ein Flashmob durch seine geschickte Internetkommunikation und die präzise Inszenierung der Aktion mit ästhetischen Mitteln aus: differenzierte Körperbewegungen an ausgewählten Orten mit Wiederholung, Einfrieren, Slow-motion oder synchronen Bewegungsabläufen sowie die Vor- und Nachbereitung im Netz. Man kann von spontanen und sozialen Choreografien im öffentlichen Raum oder öffentlichen Chören sprechen. Der Alltagskörper wird zum Kunst- und Chorkörper, die Alltagshandlung zur Kunsthandlung, der Alltagsort zum Kunstort. Neben Spaß und Action sind durch die gezielten Störungen von öffentlichen und alltäglichen Abläufen durchaus ästhetische und politische Inhalte wie Wahrnehmungsschulung, Konsumkritik und „öffentliches Sprechen" zu beobachten.

Alltagshandlung und -wahrnehmung werden gebrochen, irritiert, angehalten, überrascht und verfremdet durch die kollektive Aktion. Das Kollektiv und die relativ einfache Spielaufgabe schützt das Individuum, jede/r kann mitmachen. Der Flashmob lässt sich somit als eine Form chorischen Theaters verorten. Und wir wissen aus der Tradition des Theaterchors vom antiken Chor bis zu modernen Chören bei Ariane Mnouchkine, Einar Schleef und Volker Lösch, dass der Chor als kollektive Kraft stets ästhetisch und sozial wirksam wird (vgl. Kurzenberger 2009).

Am Flashmob lassen sich also zentrale Merkmale performativer Praxen zeigen. Die Körper-und Handlungsorientierung ist zentral, ebenso der Aufführungs- und Ereignischarakter. Aber auch Spiellust und Erlebnisintensität, Gruppenaktion und Improvisation markieren soziale und ästhetische Kriterien und Qualitäten des Performativen.

\section{Performance als Kunst, Aktion und Wirklichkeitskonstitution}

Als theoretische und begriffliche E inordnung s ei d arauf v erwiesen, $d$ ass der "performative turn" in den Kultur-, Kunst- und Sozialwissenschaften und die damit verbundene Rede vom Performativen einen Diskurs markiert, der sich aus drei Entwicklungslinien speist und die Begriffe „performativ“, „Performance“ und „Performativität" geprägt hat. Erstens: Mit der Sprechakttheorie von John Austin (1955) wird der Begriff „performativ“ als Bezeichnung für sogenanntes 
wirklichkeitskonstituierendes Sprechen eingeführt. Zweitens: „Performance“ bezeichnet seit den 1960er Jahren in den Kunst- und Theaterwissenschaften eine Kunstpraxis, die weniger ein Werk aufführt, sondern eine Aktion oder ein Ereignis ist. Drittens: In der Genderforschung wird von Judith Butler (1988) „Performativität“ als Begriff zur Bezeichnung der kulturellen Konstitution von Geschlechtsidentität (gender) durch körperliche Handlungen eingeführt (vgl. Fischer-Lichte/Kolesch/Warstat 2005, S. 231-242). Mit diesen Begriffen und Theoriebezügen wird herausgestellt, dass unser sprachliches und körperliches Handeln Wirklichkeit schaffen kann.

Mit den Begriffen Performance und postdramatisches Theater (vgl. Lehmann 1999) verbinden sich also ein verändertes Verständnis von Darstellung und Kunstpraxis. Selbst- und Rollendarstellung, Spiel und Repräsentation vermischen sich. Im Zentrum steht nicht mehr das Dramenwerk, der Text, sondern das flüchtige Ereignis der Aufführung und die ästhetische Erfahrung des Zuschauers/der Zuschauerin; nicht die Darstellung einer Rolle, sondern die Präsenz des Darstellungsaktes.

Folgende Stichworte markieren die zentralen Unterschiede und das Selbstverständnis von Performance im Vergleich zum dramatischen Theater:

- Ereignis statt Werk,

- Präsentation statt Repräsentation,

- Handeln statt Spielen und als-ob Darstellen,

- Selbstdarstellung statt Rollen- und Figurendarstellung,

- Zuschaueransprache bzw. Unmittelbarkeit statt vierter Wand und Illusion.

Aber auch die Erziehungswissenschaft beschäftigt sich zunehmend mit dem Begriff des Performativen, denn so lässt sich soziales und pädagogisches Handeln abseits von Intentionalität und Normativität anders betrachten. Die Bildungsforscher Christoph Wulf und Jörg Zirfas als Herausgeber des Bandes „Pädagogik des Performativen“ (2007) weisen mit Nachdruck auf die Bedeutung performativer Erfahrungs- und Bildungsprozesse hin, denn diese werden nicht verbal vermittelt, sondern körperlich inszeniert und aufgeführt. Pädagogisches Handeln ist somit auch als Arbeit mit und am menschlichen Körper zu verstehen, was in der Schule außer im Sport vorwiegend im Theaterspiel möglich ist. Das heißt, die Inszenierung und Aufführung des Körpers ist elementar für Bildungsprozesse und muss entsprechend gestaltet und reflektiert werden. Eine Orientierung an den Körper-Künsten (Theater, Tanz, Performance) und deren performativer Praxen ist daher für pädagogische bzw. didaktische Lernkonzepte und Praxismodelle sinnvoll und notwendig. 


\section{TheaterSprachCamp Hamburg}

Das TheaterSprachCamp Hamburg existiert seit 2007 und hat mittlerweile fünfmal stattgefunden. Es ist ein erfolgreiches, permanent verbessertes und evaluiertes Modell zur ganzheitlichen Sprachförderung, das hier als Lernen mit und durch Theater vorgestellt werden soll.

„Irgendwann stellt sich die Frage: Bleibt es bei der Spracherwerbsanstrengung oder verliebt man sich?" Der deutschtürkische Schriftsteller und Dramatiker Feridun Zaimoglu (2008) ${ }^{1}$ macht hier deutlich, dass Spracherwerb immer auch eine soziale Komponente hat.

Spracherwerb muss keinen Widerspruch zum wirklichen Leben darstellen. Im Gegenteil, das Ziel des Hamburger TheaterSprachCamps ist es Sprachförderung lebensnah und spielerisch im Kontext einer Ferienfreizeit zu vermitteln. In dem dreiwöchigen Freizeitcamp gehören deshalb Sprachförderung, Theaterund Freizeitaktivitäten als gemeinsames Leben und Lernen selbstverständlich zusammen. Und nur so erleben die Kinder Sprache und Sprechenlernen als eine positive im sozialen und ästhetischen Tun angelegte Lernerfahrung.

\subsection{Die Eckdaten des Camps}

Die Umsetzung der TheaterSprachCamps ist mit organisatorischen, logistischen und kommunikativen Herausforderungen verbunden. Die Eckdaten vermitteln davon einen Eindruck:

- 3 Wochen Ferienfreizeit,

- 280 Kinder der dritten und vierten Klasse mit einfachem und z.T. besonderem Sprachförderbedarf und Migrationshintergrund,

- 70 Mitarbeiter: 20 Studierende für den Bereich Sprachförderung, 20 Studierende für den Bereich Theaterpädagogik, 30 Mitarbeiter unterschiedlichen Alters für den Bereich Freizeitpädagogik,

- 10 Standorte bzw. Freizeitheime im Hamburger Umland und auf der Insel Sylt: je Standort 28 Kinder eingeteilt in zwei Gruppen mit je 14 Kindern,

- 7 Mitarbeiter (2 Sprach-, 2 Theater-, 3 Freizeitpädagogen) pro Team für 28 Kinder,

- 300 000.- $€$ Gesamtetat (Konzeptentwicklung, Schulung, Freizeitmaßnahme, Mitarbeiterhonorare, Material, gemeinsames Abschlussfest in Hamburg),

- Kooperationsprojekt der Schulbehörde, der Universität, des Landesinstituts für Lehrerbildung, des Jugenderholungswerks, der academie creatar.

\footnotetext{
${ }^{1}$ Vgl. Frankfurter Rundschau vom 21.2.2008, S. 34.
} 
Grundlegend für das Hamburger Konzept ist das produktive Ineinandergreifen der drei Bereiche Sprache, Theater und Freizeit. Das integrative Lern- und Übungsprogramm in den Bereichen Sprache und Theater wird Werkstatt genannt. An fünf Tagen in der Woche finden vier Stunden gemeinsame Werkstatt und eine Stunde (Vor)Lesen statt.

Das übergeordnete Lernziel der Camparbeit liegt in der Entwicklung einer positiven Einstellung der Kinder zum eigenen Lernvermögen. Sie sollen sich spielerisch mit Sprache und Texten auseinandersetzen und dabei gleichzeitig den Gebrauchswert von Lesen und Grammatik erfahren. Übertragen auf die Schule sollte sich über das Camp hinaus die Unterrichtssprache verbessern und insbesondere das im Camp Gelernte in der Schule aktiv zur Anwendung kommen. Die Herkunftssprachen der Kinder werden dabei explizit berücksichtigt, denn die Zwei- und Mehrsprachigkeit der Kinder wird als positive Fähigkeit und Lernchance angesehen. Jenseits ihrer zum Teil schwierigen häuslichen Situationen und prekären sozialen Lagen erfahren die Kinder im Camp eine allgemeine Wertschätzung in der Gruppe und erleben dies als Empowerment.

Das Konzept des Hamburger TheaterSprachCamps wird unter universitärer Leitung (Theaterpädagogik / Sprachförderung, DaZ) jährlich evaluiert und modifiziert (vgl.www.epb.uni-hamburg.de/de/personen/neumann).Zudem gilt es im Leitungsteam mit allen beteiligten Organisationen (s.o.) die Realisierung und Evaluation des Projektes zu koordinieren; die Vorbereitung und Schulung der Studierenden als Betreuer findet ebenfalls im universitären Rahmen statt.

\subsection{Theaterarbeit}

Warum ist eine Verbindung von Theaterspielen und Sprache, Sprechen, Sprachförderung sinnvoll, produktiv und Erfolg versprechend? Theaterarbeit wird nie nur als Methode verstanden, sondern sie ist im Sinne eines erweiterten Theaterverständnisses (Performance) performatives und künstlerisches Handeln und kann somit als grundständige ästhetische Bildung bezeichnet werden.

Theater und Sprache gehören im künstlerischen Genre des Sprechtheaters elementar zusammen. Sprache und Sprechen sind neben dem Körper zentrales Ausdrucks- und Gestaltungsmittel der szenischen Aktion und der theatralen Kommunikation. Theater arbeitet mit Sprache und fördert daher das Sprechenwollen. Darüber hinaus spricht das Theaterspiel die beteiligten Spieler ganzheitlich an und setzt über seine Spiel- und Interaktionsformen soziale Prozesse und Selbstwahrnehmungsprozesse in Gang.

Theater hat darüber hinaus verschiedene Merkmale, die es als Lern- und Kommunikationsform auszeichnen:

- Theater ist unmittelbare Live-Kommunikation von Mensch zu Mensch.

- Theater ist eine soziale Kunstform, es entsteht nur im gemeinsamen Miteinander.

- Im Theater ist der Mensch mit seinen Ausdruckmöglichkeiten immer Gestalter und Gestaltungsmaterial zugleich 
- Theater thematisiert meist Fragen oder Aspekte des menschlichen (Zusammen)Lebens.

- Theater verlangt eine Auseinandersetzung mit sich selbst und anderen, weil man zumindest körperlich anwesend ist.

- Die Verbindung von Theater und Sprache ist also nahe liegend.

Drei Lernebenen des Theaterspielens sind für die theaterpädagogische Arbeit und die gemeinsame Werkstatt von Theater und Sprache zu unterscheiden und einzubinden.

- Soziales und emotionales Lernen

- Fachliches und themenorientiertes Lernen

- Ästhetisches Lernen und Kommunizieren

Diesen Lernebenen lassen sich entsprechenden Lernziele und theaterpädagogischen Inhalte zuordnen.

\section{Soziales und emotionales Lernens heißt Lernvoraussetzungen zu schaffen.}

Es gilt Konzentration, Atmosphäre, Entspannung und Motivation für individuelle, soziale und sprachliche Wahrnehmungs- und Lernprozesse herzustellen. Theaterspielen als soziale Interaktionsform verfügt über einen reichen Fundus an Spielen, die eine Gruppe durch Bewegung, Spielaufgaben, Rhythmus oder Lieder gemeinsam und einzeln agieren lassen. Erstes Lernziel ist es Voraussetzungen und Möglichkeiten der Begegnung und des sich Kennenlernens in der Gruppe zu schaffen. Es geht darum über die Spiellust ein Klima der Offenheit und Angstfreiheit zu schaffen. Weitere Lernziele bestehen darin, dass die Kinder mal durch Entspannung, mal durch Anspannung/Auspowern innerlich ankommen und ruhig werden: Konzentration und gegenseitige Wahrnehmung und Achtung.

Als Anfangsformation für Gruppenübungen bietet sich der Kreis an. Andere Sozialformen in der Theaterarbeit neben Kreis und Gegenüberstehen sind Einzel-, Partner- und Gruppenarbeit. Die Bedeutung des Kreises ist vielfältig: alle sehen einander, alle stehen gleich berechtigt nebeneinander, alle sind beteiligt. Im Kreis lassen sich unterschiedliche Spielformen und Aktivitäten einüben. Imitation: Nachmachen, was Spielleiter oder einzelne vormachen; Impuls: durch den Nachbarn Bewegungs-, Sprech-, Tonimpulse weitergeben; Kollektiv: alle agieren gemeinsam als Gruppe. Der Kreis eignet sich auch für Anfangs- und Abschlusseinheiten der Gruppenarbeit und Rituale. 


\section{Fachliches und themenorientiertes Lernen heißt im Theater Wahrnehmung, Ausdruck und Zusammenspiel zu trainieren.}

Auch im TheaterSprachCamp geht es zunächst darum, die Grundlagen der szenischen Arbeit kennen zu lernen und einzuüben. Theaterspielen als eine ganzheitliche - mal spontane, mal gestaltete - Form des Sprechens vor anderen mit Stimme, Sprache, Körper, Bewegung, einzeln und in der Gruppe ist immer Sprach- und Ausdruckschulung. Das Sprechen findet dabei auf unterschiedlichen Ebenen statt; im Theater unterscheiden wir folgende „Sprachen“ und Sprechformen:

- der Sprache des Körpers, des Raums, der Gruppe,

- mit Sprechen im Dialog, Monolog und Chor,

- mit Erzählen, Spielen und Interpretieren von Geschichten.

Einzel-, Partner- und Gruppenübungen vermitteln gezielt Grundfertigkeiten wie Körperspannung, Artikulation, Aktion-Reaktion, Impulse geben und nehmen. Die Übungen dienen der Selbst- und Fremdwahrnehmung und der kontinuierlichen Einübung von Ausdruck, Präsenz und Zusammenspiel, d.h. die Übungen sind wie ein Training zu wiederholen und zu steigern. Einzelne Schwerpunkte können den Umgang mit Atem und Stimme oder den Umgang mit Zeit und Raum, Material und Requisiten thematisieren. Spiegelübungen mit Partnern trainieren das genaue Wahrnehmen, das Vor- und Nachmachen.

\section{7 Ästhetisches Lernen und Kommunizieren heißt theatrales Spielen und Sprechen zu vermitteln.}

Auf dieser Ebene fängt die szenische Arbeit an, es werden die ersten Körperbilder und Szenen gebaut. Einzelne Gestaltungselemente des Theaters (Text, Figur, Situation, Handlung, Raum, Musik) werden ausprobiert, entwickelt und zusammengesetzt. Dazu kommt es über Improvisation und Spielaufgaben zu ersten gemeinsamen Spielszenen. Wörter, Sätze und Bilder können in Szene gesetzt werden bis hin zur szenischen Umsetzung eines Kinderbuches.

\section{Evaluation}

Das Hamburger TheaterSprachCamp wird vom Landesinstitut für Lehrerbildung und Schulentwicklung kontinuierlich evaluiert, die einzelnen Testverfahren, Evaluationsdetails und Ergebnisse können hier nicht im Detail ausgeführt werden (vgl. dazu den Beitrag von Julia Kinze in dieser Ausgabe). Allgemeine Ergebnisse lassen sich jedoch so zusammenfassen: Schülerinnen und Schüler, 
die im TheaterSprachCamp betreut wurden, weisen im Vergleich mit einer nicht betreuten Kontrollgruppe deutlich höhere Lernzuwächse auf. Diese Lernerfolge zeigen sich besonders in der Lesefertigkeit, im Bereich der Wortschatzerweiterung, des Sprachflusses, aber auch in einem Zuwachs an grammatikalischen Kenntnissen. Die Aussagen der Schülerinnen und Schüler selbst und ihrer Eltern in den Befragungen nach dem Camp zeigen, dass die Beteiligten mit dem TheaterSprachCamp nicht nur in sehr hohem Maße zufrieden sind, sondern auch eine deutliche Verbesserung der Lerneinstellung festzustellen ist. Über das Theaterspiel und das soziale Miteinander erhalten die Kinder ein deutlich höheres Selbstbild und gestärktes Selbstbewusstsein. Sprachförderung im Rahmen des TheaterSprachCamp ist in dieser Form somit nicht nur eine Schule des Sprechens, sondern auch eine Schule des Handelns, der intensiven Wahrnehmung und Begegnung und somit letztlich eine Schule der Integration und Partizipation.

Festzustellen ist abschließend jedoch, dass es ein Problem der Nachhaltigkeit gibt, denn über den Zeitraum eines Jahres schwächen sich die Lernerfolge nach und nach ab. Dieser Effekt ist bei solchen kurzzeitigen intensiven Interventionen bekannt. Im Hinblick auf eine verbesserte Nachhaltigkeit ist es daher dringend geboten Folgemaßnahmen zu entwickeln, die im (Unterricht des) laufenden Schuljahr/es implementiert werden können, um die positiven Effekte zu festigen. So sind als erste Maßnahme vier ganztägige Projekttage mit einem Werkstattprogramm (vergleichbar dem des TheaterSprachCamps) an einem außerschulischen Lernort und/oder Nachmittagseinheiten an ausgewählten Ganztagesschulen geplant.

\section{Bibliografie}

Enzensberger, Hans Magnus (1987): Ach Europa! Wahrnehmungen aus sieben Ländern. Frankfurt/Main: Suhrkamp

Fischer-Lichte, Erika (2004): Ästhetik des Performativen. Frankfurt/Main: Suhrkamp

Fischer-Lichte, Erika et al. (Hrsg.) (2005): Metzler Lexikon Theatertheorie. Stuttgart/Weimar: Metzler

Kinze, Julia (2012): Das Hamburger TheaterSprachCamp: Methoden und Ergebnisse der Evaluation. In: Scenario VI, 1, 2012, 85-101

Klein, Gabriele; Sting, Wolfgang (Hrsg.) (2005): Performance. Positionen zur zeitgenössischen szenischen Kunst. Bielefeld: Transcript

König, M. (2009): ,Yeah` - das letzte Mittel gegen Merkel. In: Süddeutsche Zeitung [www.sueddeutsche.de/politik/flashmobs-im-wahlkampf-yeahdas-letzte-mittel-gegen-merkel-1.42062; 10.5.2012]

Kurzenberger, Hajo (2009): Der kollektive Prozess des Theaters. Chorkörper, Probengemeinschaften, theatrale Kreativität. Bielefeld: Transcript 
Lehmann, Hans-Thies (1999): Postdramatisches Theater. Frankfurt/Main: Verlag der Autoren

Liebau, Eckhart; Klepacki, Leo; Zirfas, Jörg (2009): Theatrale Bildung. Theaterpädagogische Grundlagen und kulturpädagogische Perspektiven für die Schule. Weinheim/München: Beltz-Juventa

McKenzie, Jon (2001): Perform or Else. From Discipline to Performance. London: Routledge, Chapman \& Hall

Neumann, Ursula et al. (2011): Hamburger TheaterSprachCamp 2011.

[http://www.epb.uni-

hamburg.de/files/u14/Konzept_TSC_2011_final.pdf/; 1.5.2012]

Sting, Wolfgang (2010): Theater und Sprache. Das Hamburger TheaterSprachCamp aus theaterpädagogischer Sicht. In: Sting, W. et al. (Hrsg.): Irritation und Vermittlung. Theater in einer interkulturellen und multireligiösen Gesellschaft. Berlin: Lit, 203-214

Sting, Wolfgang (2011): „Und alle so: Yeaah“ Performance als Experimentierund Freiheitsraum. In: Bockhorst, H. (Hrsg.) KUNSTstück FREIHEIT. München: Kopäd, 172-178

Wulf, Christoph; Zirfas, Jörg (Hrsg.) (2007): Pädagogik des Performativen. Theorien, Methoden, Perspektiven. Weinheim/Basel: Beltz 